\title{
1s3p Resonant Inelastic X-ray Scattering of Cobalt Oxides and Sulfides
}

\author{
Mustafa Al Samarai, ${ }^{\dagger}$ Mario Ulises Delgado-Jaime, ${ }^{\dagger}$ Hirofumi Ishii, $^{\ddagger}$ Nozomu Hiraoka, ${ }^{\ddagger}$ Ku-Ding Tsuei, ${ }^{\ddagger}$ \\ Jean-Pascal Rueff, ${ }^{\S}$ Benedikt Lassale-Kaiser, ${ }^{\S}$ Bert M. Weckhuysen, ${ }^{\dagger}$ and Frank M.F. de Groot ${ }^{*}, \dagger$ \\ ${ }^{\dagger}$ Inorganic Chemistry and Catalysis, Debye Institute for Nanomaterials Science, Utrecht University, Universiteitsweg 99,3584 CG \\ Utrecht, Netherlands \\ ${ }^{\ddagger}$ National Synchrotron Radiation Research Center, Hsinchu Science Park, Hsinchu 30076, Taiwan \\ ${ }^{\S}$ Synchrotron SOLEIL, L'Orme des Merisiers, Saint-Aubin, BP 48, 91192 Gif-sur-Yvette Cedex, France
}

ABSTRACT: We are developing $1 \mathrm{~s} 3 \mathrm{p}$ resonant inelastic X-ray scattering (RIXS) as a versatile spectroscopic tool to probe active systems under working conditions. In order to establish detailed reference materials for cobalt-based systems, we have applied 1s X-ray absorption spectroscopy (XAS) and 1s3p RIXS to cobalt oxides (CoO, $\left.\mathrm{Co}_{3} \mathrm{O}_{4}\right)$ and sulfides $\left(\mathrm{CoS}\right.$ and $\left.\mathrm{CoS}_{2}\right)$. We show that the $\mathrm{Co}^{3+}$ ions in $\mathrm{Co}_{3} \mathrm{O}_{4}$ contain a large nonlocal peak intensity in the pre-edge and that the resonant $3 \mathrm{p}$ final state of $\mathrm{Co}_{3} \mathrm{O}_{4}$ is dominated by the two exchangesplit peaks of the tetrahedral $\mathrm{Co}^{2+}$ site. In contrast, the $3 \mathrm{p}$ final state of $\mathrm{CoS}$ and $\mathrm{CoS}_{2}$ shows a single asymmetric peak due to the large screening of the 3p-3d exchange interaction. While $\mathrm{CoS}$ has a single pre-edge peak, $\mathrm{CoS}_{2}$ contains an additional large nonlocal peak. This indicates that although $\mathrm{CoS}_{2}$ is often indicated as $\mathrm{Co}^{2+}\left(\mathrm{S}_{2}\right)^{2-}$, it has a very strong sulfur-mediated cobalt-cobalt bonding, similar to the low-spin $\mathrm{Co}^{3+}$ sites in oxides. These electronic structure details reveal the significance of $1 \mathrm{~s} 3 \mathrm{p}$ RIXS spectroscopy for determining the symmetry, spin and covalence of transition metal compounds, information that is largely inaccessible in conventional K edge XAS. The experimental results are simulated with multiplet calculations that reveal the partial screening of the $3 \mathrm{p} 3 \mathrm{~d}$ exchange interaction and the presence of nonlocal transitions. We conclude that 1s 3 p RIXS combined with spectral simulations presents a valuable tool to unravel the electronic properties of functional materials under working conditions.

\section{INTRODUCTION}

Recent developments in theoretical and experimental resonant inelastic X-ray scattering (RIXS) studies have allowed the application of this technique in various fields of chemistry, including heterogeneous catalysis. ${ }^{1,2}$ The K-edge of $3 \mathrm{~d}$ transition metal ions relates to $1 \mathrm{~s} \rightarrow 4 \mathrm{p}$ excitations and reveals information about the oxidation state and the neighboring ligands. The pre-edge resulting from the $1 \mathrm{~s} \rightarrow 3 \mathrm{~d}$ quadrupole transition contains additional information, including the symmetry around the transition metal ion and its spin state. $^{3,4}$ Due to the $1 \mathrm{~s}$ core-hole lifetime, the K-edges are broadened with a Lorentzian of approximately $1.5 \mathrm{eV}$ and so the high intensity of the main K-edge $(1 s \rightarrow 4 p)$ peak and its large broadening creates a background around the pre-edge structure. The origin of the pre-edge feature may be due to three, interconnected, effects:

1. A direct $1 \mathrm{~s} \rightarrow 3 \mathrm{~d}$ quadrupole transition that can be calculated as a quadrupole transition from $3 \mathrm{~d}^{n}$ to $1 \mathrm{~s}^{1} 3 \mathrm{~d}^{n+1}$.

2. If centro-symmetry is broken, the metal $4 p$ states mix with the $3 \mathrm{~d}$ states, allowing dipole transitions into the pre-edge that have more intensity than the correspond- ing quadrupole intensity. The dipole spectral intensity can be up to $50 \times$ stronger than the quadrupole intensity.

3. If a short cobalt-oxygen-cobalt moiety is present additional dipole transitions into the $3 \mathrm{~d}$-band can be observed related to the overlap of cobalt $4 p$ states with cobalt $3 \mathrm{~d}$ states of near-neighbors. Because these socalled nonlocal states are less affected by the core hole in the final state they appear at approximately $3 \mathrm{eV}$ higher incident energies. 3,5

After the excitation of a 1s electron, the subsequent radiative decay with the highest probability is a $1 \mathrm{~s} 2 \mathrm{p}(\mathrm{K} \alpha)$ transition, while the $1 \mathrm{~s} 3 \mathrm{p}(\mathrm{K} \beta)$ transitions are theoretically a factor 6-8 weaker. ${ }^{6}$ At the pre-edge resonance, the $\mathrm{K} \alpha$ decay involves a transition from the $1 \mathrm{~s}^{1} 3 \mathrm{~d}^{n+1}$ to the $2 \mathrm{p}^{5} 3 \mathrm{~d}^{n+1}$ states, which is an identical final state configuration as in the soft $\mathrm{X}$-ray $\mathrm{L}_{2,3}$-edges. Equivalently, $\mathrm{K} \beta$ decay reaches the same $3 \mathrm{p}^{5} 3 \mathrm{~d}^{n+1}$ final states as the $\mathrm{M}_{2,3}$-edges XAS. While $2 \mathrm{p}^{5} 3 \mathrm{~d}^{n+1}$ final states are dominantly split by the $2 \mathrm{p}$ spin-orbit splitting, the $3 \mathrm{p}^{5} 3 \mathrm{~d}^{n+1}$ states are split

Received: June 25, 2016

Revised: October 8, 2016

Published: October 10, 2016 

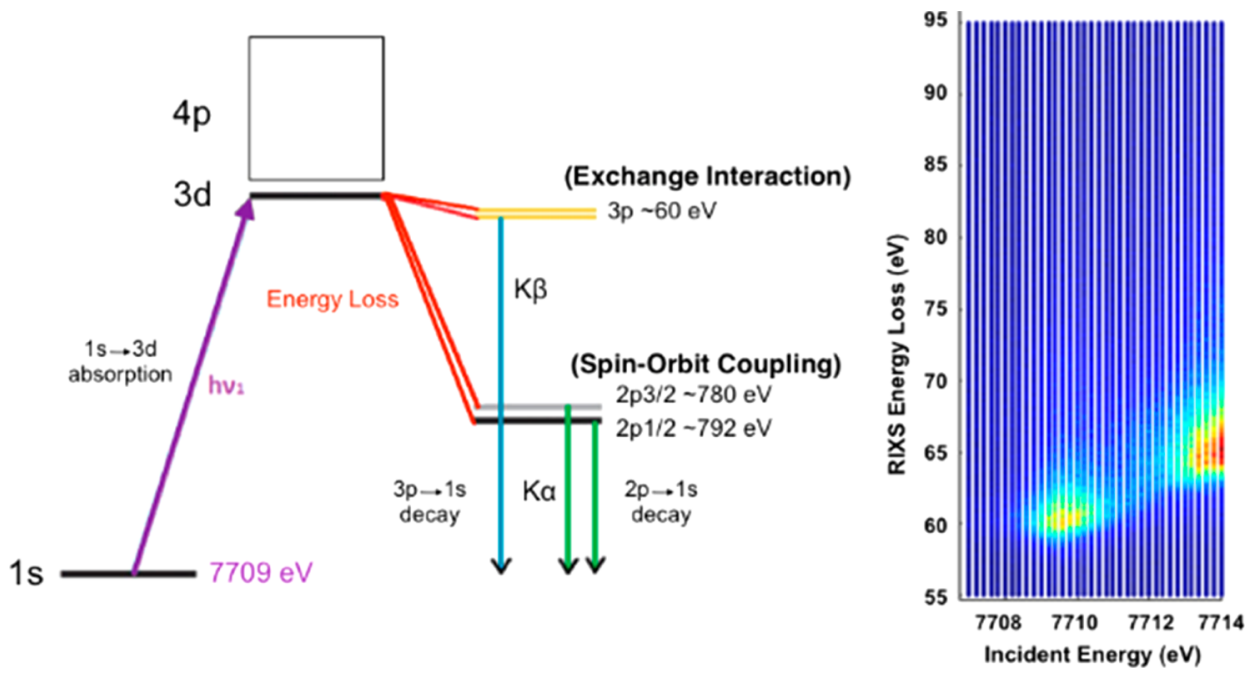

Figure 1. Main transitions and interactions involved in $1 \mathrm{~s} 2 \mathrm{p}$ and $1 \mathrm{~s} 3 \mathrm{p}$ RIXS processes for cobalt-containing compounds (left). A typical RIXS 2D plane consisting of multiple emission slices measured at various incident energies (right). The RIXS slices are then interpolated to obtain a full RIXS 2d plane.

by the large 3p3d exchange interactions, which implies that the $\mathrm{K} \beta$ emission spectrum is sensitive to the intra-atomic exchange interactions and as such to the local moment on the $3 \mathrm{~d}$ states. In general, the $3 \mathrm{~d}$ transition metal (TM) $\mathrm{K} \beta$ emission spectrum consists of the main emission line called the $K \beta_{1,3}$, a relatively broad $\mathrm{K} \beta^{\prime}$ peak, and very weak emission band $\mathrm{K} \beta_{5}$ (valence-tocore emission). The $\mathrm{K} \beta^{\prime}$ line originates from the exchange interaction between the electrons in the $3 \mathrm{~d}$ shell and the hole in the $3 p$ shell due to the emission of the $\mathrm{K} \beta_{1,3}$ line. Consequently, the energy separation between the $K \beta^{\prime}$ and $K \beta_{1,3}$ lines and the intensity ratio of the $K \beta^{\prime}$ to $K \beta_{1,3}$ lines depend on the number of the unpaired $3 \mathrm{~d}$ electrons. Due to the correlation between the number of the unpaired $3 \mathrm{~d}$ electrons to these band structures some interaction is expected between the features of the $\mathrm{K} \beta^{\prime}$ line and the valence- and conduction-band structures of $3 \mathrm{~d}$ transition metal elements.

The 1s3p RIXS experiment involves a photon-in-photonout process in which the incident energy and emitted energy are varied, yielding a two-dimensional spectral surface. To represent the results, the excitation energy $\left(E_{\text {in }}\right)$ is set as the horizontal axis, while the vertical axis is either set as the emission energy $\left(E_{\text {out }}\right)$ or as the energy loss energy $\left(E_{\text {in }}-E_{\text {out }}\right)$.

Figure 1 is an illustration of the $1 \mathrm{~s} 2 \mathrm{p}$ and $1 \mathrm{~s} 3 \mathrm{p}$ RIXS processes for $3 \mathrm{~d}$ cobalt compounds. At $7709 \mathrm{eV}$ the $1 \mathrm{~s}$ to $3 \mathrm{~d}$ quadrupole transition is visible. The vertical axis represents the energy loss $\left(E_{\text {in }}-E_{\text {out }}\right)$ and effectively shows the $3 p$ final state binding energy at approximately $60 \mathrm{eV}$. In addition, the onset of the main K edge is expected at slightly higher energy $(\sim 7714$ $\mathrm{eV}$ ) and its $1 \mathrm{~s} 3 \mathrm{p}$ fluorescence at $65 \mathrm{eV}$. The vertical lines (indicated $\mathrm{K} \beta$ and $\mathrm{K} \alpha$ ) indicate the actual emission measurements that are taken at fixed excitation energies. We note that the pre-edge feature (related to the $3 \mathrm{~d}$-states) is separated by $\sim 4 \mathrm{eV}$ from the edge intensity. Thus, by measuring a vertical slice at the pre-edge energy, the $1 s^{1} 3 \mathrm{~d}^{n+1}$ to $3 \mathrm{p}^{5} 3 \mathrm{~d}^{n+1}$ resonant features are probed. Moreover, the diagonal line in a RIXS energy loss map yields the so-called High Energy Resolution Fluorescence Detection (HERFD) spectrum, which in a first approximation is similar to a sharpened version of the $\mathrm{K}$ edge absorption spectrum. ${ }^{8,9}$

In this study we have performed 1s $3 p$ RIXS measurements on two cobalt oxides (i.e., $\mathrm{CoO}$ and $\mathrm{Co}_{3} \mathrm{O}_{4}$ ) and two cobalt sulfides (i.e., $\mathrm{CoS}$ and $\mathrm{CoS}_{2}$ ) that serve as reference materials for the evolution of cobalt species within the industrially relevant cobalt(nickel)-molybdenum-sulfide $(\mathrm{Co}(\mathrm{Ni}) \mathrm{MoS})$ hydro-desulphurization (HDS) catalyst system, known to remove sulfur impurities from crude oil-derived hydrocarbon feedstocks. $^{10}$

The local structure, symmetry, and spin state of the $\mathrm{Co}_{3} \mathrm{O}_{4}$, $\mathrm{CoO}, \mathrm{CoS}$, and $\mathrm{CoS}_{2}$ samples are summarized in Figure 2. We

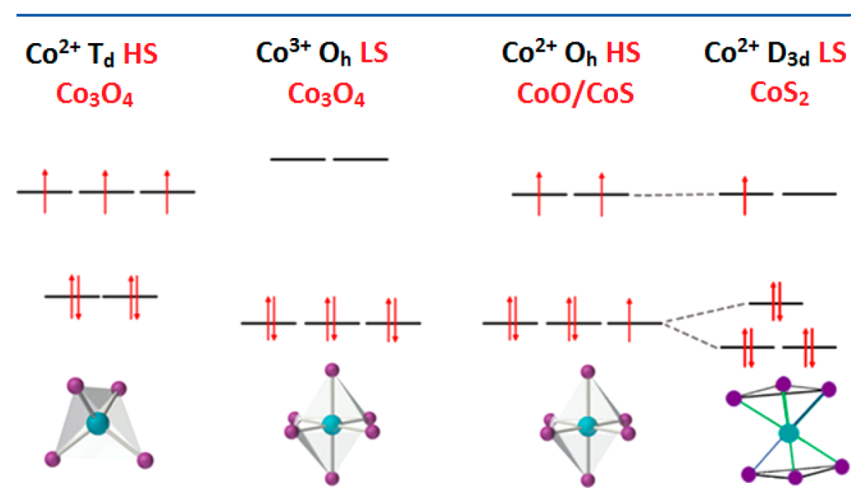

Figure 2. Local structures, proposed symmetries and corresponding crystal field diagrams for $\mathrm{Co}_{3} \mathrm{O}_{4}$ (which is a mixed valence material with $\mathrm{Co}^{2+} T_{\mathrm{d}}$ high-spin), and a $\mathrm{Co}^{3+} \mathrm{O}_{\mathrm{h}}$ low-spin, high-spin $\mathrm{O}_{\mathrm{h}} \mathrm{CoO}$, high-spin $\mathrm{CoS}$ and low-spin $\mathrm{D}_{3 d} \mathrm{CoS}_{2}$.

note here that an additional sulfur in $\mathrm{CoS}_{2}$, which distorts the symmetry from octahedral in $\operatorname{CoS}$ to $D_{3 d}$, causes the spin to change from high to low spin. There have been several studies in the literature reporting relevant information regarding the properties of cobalt oxide compounds. ${ }^{11-14}$ Furthermore, the oxygen mediated $\mathrm{d}-\mathrm{d}$ interactions are also reported to affect the electronic properties of several transition metal oxides.

\section{EXPERIMENTAL SECTION}

2.1. Materials. $\mathrm{CoO}$ was obtained from SPL.EU crystals as a single crystal of $\mathrm{CoO}(001)$, while $\mathrm{Co}_{3} \mathrm{O}_{4}$ was supplied from Sigma-Aldrich (99.99\% trace metal basis). Cobalt sulfides were supplied from Alfa-Aesar as CoS (99.5\% on metal basis) and $\mathrm{CoS}_{2}$ (99.5\% on metal basis). 
2.2. X-ray Experiments. The experiments with cobalt oxides were performed at the Taiwan beamline BL12XU at the Spring-8 synchrotron facilities. ${ }^{15}$ The optical system consists of four major elements, a high heat-load Si (111) double-crystal premonochromator, a cylindrical collimating mirror, a channelcut high-resolution monochromator, and a toroidal focusing mirror. The incident energy was selected using the Si (400) reflection during the measurements at the Co K-edge. After the monochromator, the beam is delivered to the Pt-coated focusing mirror and focused both vertically and horizontally to $\sim 80(\mathrm{~V}) \times 120(\mathrm{H}) \mu \mathrm{m}^{2}$ at the sample position. The sample, crystal analyzer and a photon detector were arranged in a horizontal Rowland geometry. The Co K $\beta$ (1s3p) RIXS spectra were recorded at a scattering angle of $90^{\circ}$ in the horizontal plane using a $1 \mathrm{~m}$ radius spherically bent $\mathrm{Ge}$ (444) analyzer. The total energy resolution of the monochromator (photon-in) and the spectrometer (photon-out) is approximately $0.4 \mathrm{eV}$. The intensity was normalized to the incident flux. The RIXS data are shown as a contour map in a plane of incident and transferred photon energies, where the vertical axis represents the energy difference between the incident and emitted energies (energy loss). The variations of the color on the plot correspond to the different scattering intensities.

The cobalt sulfide reference samples were measured at the GALAXIES beamline at the SOLEIL synchrotron. ${ }^{16}$ Both the cobalt sulfide samples were kept under a nitrogen atmosphere during the measurements. The incident energy is selected by a Si (111) double crystal monochromator, using the third order harmonic with an energy resolution of $200 \mathrm{meV}$. The beam is focused both vertically and horizontally to $\sim 30(\mathrm{~V}) \times 80(\mathrm{H})$ $\mu \mathrm{m}^{2}$ at the sample position using a toroidal mirror located downstream. The spectra were recorded at $45^{\circ}$ in the vertical plane using the beamline RIXS spectrometer and a spherically bent $\mathrm{Ge}$ (111) analyzer. The total energy resolution of the beamline and the spectrometer is $\sim 0.4 \mathrm{eV}$. The intensity was normalized to the incident flux. The RIXS 2D maps were obtained in the excitation range of $7700-7750 \mathrm{eV}$ with steps of $0.25 \mathrm{eV}$, and 57.5 to $75 \mathrm{eV}$ for the RIXS energy loss, with steps of $0.25 \mathrm{eV}$. The emission spectrometer energy resolution was measured by taking the full width at half-maximum (fwhm) of the elastic peak at $7720 \mathrm{eV}$. Radiation damage studies were performed at ambient conditions by measuring three XAS spectra to check for any shifts in the edge jump energy, and the pre-edge intensity and energy shift. Additionally, six RIXS spectra were measured to reconfirm the results obtained with XAS. For all the reference spectra, there was no change in the measured spectra for a $3 \mathrm{~h}$ experiment, confirming the absence of radiation damage.

2.3. Theory. We have applied crystal-field multiplet theory and charge-transfer multiplet theory to calculate the 1s XAS pre-edge and 1s3p RIXS spectral shapes. The pre-edge in the Kedge XAS of $\mathrm{Co}^{2+}$ in $\mathrm{CoO}$ and $\mathrm{CoS}$ has been calculated as the quadrupole transition from $3 \mathrm{~d}^{7}$ to $1 \mathrm{~s}^{1} 3 \mathrm{~d}^{8}$ in octahedral symmetry. In the case of $\mathrm{Co}_{3} \mathrm{O}_{4}$, for the tetrahedral $\mathrm{Co}^{2+}$, the dipole channel from $3 \mathrm{~d}^{7}$ to $1 \mathrm{~s}^{1} 3 \mathrm{~d}^{7} 4 \mathrm{p}^{1}$ has been added to the direct quadrupole transitions to the corresponding charge transfer multiplet calculations. ${ }^{17-22}$ In all cases, the 1s3p X-ray emission channel is calculated as the transition from $1 s^{1} 3 d^{8}$ to $3 \mathrm{p}^{5} 3 \mathrm{~d}^{8}$ and to generate the 1s $3 p$ RIXS plane, the excitation and decay are coupled using the Kramers-Heisenberg equation. ${ }^{23}$

To perform these calculations, we have made use of the CTM4XAS and CTM4RIXS graphical user interfaces with the exception of the charge transfer calculation involving dipole- quadrupole mixing, which has been performed separately. ${ }^{24}$ The parameters used here for $\mathrm{CoO}$ were taken from previous studies. ${ }^{25}$ The Slater integrals were set to $90 \%$ of their atomic values to account for charge transfer effects and the crystal field (10 Dq) was set to $1.05 \mathrm{eV}$ (for $\mathrm{CoO}$ ) for the initial state and $0.9 \mathrm{eV}$ for the intermediate and final states to compensate for the effects from the $1 \mathrm{~s}$ and $3 \mathrm{p}$ core holes.

\section{RESULTS AND DISCUSSION}

3.1. Cobalt Oxides 1s3p RIXS Planes. Figure 3 shows the experimental Co K $\beta$ RIXS energy loss (EL) maps over the pre-

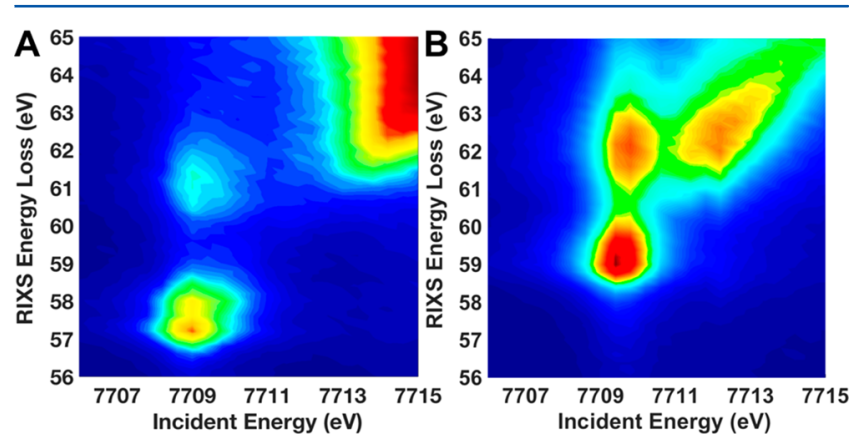

Figure 3. Experimental Co 1s3p RIXS plane of (A) $\mathrm{CoO}$ and (B) $\mathrm{Co}_{3} \mathrm{O}_{4}$.

edge region of $\mathrm{CoO}$ and $\mathrm{Co}_{3} \mathrm{O}_{4}$. As was shown by Kurian et al. for $\mathrm{CoO},{ }^{10}$ the comparison with the conventional X-ray absorption spectrum shows how the pre-edge structure is better separated from the main edge in the RIXS spectrum.

The CoO pre-edge shows two energy loss features at 58.5 and $62.0 \mathrm{eV}$ for the incident energy of $7709 \mathrm{eV}$. As we will show below, these peaks are due to the exchange splitting in the $3 \mathrm{p}^{5} 3 \mathrm{~d}^{7}$ final state. $\mathrm{Co}_{3} \mathrm{O}_{4}$ is a mixed-valence system that consists of high-spin tetrahedral $\mathrm{Co}^{2+}$ and low-spin octahedral $\mathrm{Co}^{3+}$ sites. The RIXS map shows two energy loss peaks at 59 and 62 $\mathrm{eV}$ for the $7709.4 \mathrm{eV}$ incident energy. ${ }^{26}$ These peaks are equivalent to the situation in $\mathrm{CoO}$ and we assign them to be due to the strong exchange splitting in the final state of the high-spin tetrahedral $\mathrm{Co}^{2+}$. In addition, the $\mathrm{Co}^{3+}$ energy loss feature is expected to be at $62 \mathrm{eV}$ merged with that of $\mathrm{Co}^{2+}$ emission. Furthermore, there is also a third peak visible in $\mathrm{Co}_{3} \mathrm{O}_{4}$ at an excitation energy of $7712.7 \mathrm{eV}$. The energy difference of $2.3 \mathrm{eV}$ is too large to be related to the difference between $\mathrm{Co}^{2+}$ and $\mathrm{Co}^{3+}$ sites. Instead, this feature can be assigned to a nonlocal peak often observed in low-spin $\mathrm{Co}^{3+}$ systems, and has been explained in detail elsewhere for the case of $\mathrm{LiCoO}_{2}{ }^{27}$ This nonlocal peak relates to a dipole transition from a $1 \mathrm{~s}$ core electron to a $\mathrm{Co}^{3+} 4 \mathrm{p}$ orbital that interacts with a cobalt-based $3 \mathrm{~d}$ orbital via the bridging oxygen $2 \mathrm{p}$ contribution. We note also that the local peak is often much lower in intensity than the nonlocal. ${ }^{28,29}$

3.2. Cobalt Sulfides 1s3p RIXS Planes. Figure 4 shows the experimental Co K $\beta$ RIXS energy loss (EL) maps over the pre-edge region of $\mathrm{CoS}$ and $\mathrm{CoS}_{2}$. The $\mathrm{CoS}$ pre-edge shows a single peak at $7709 \mathrm{eV}$ excitation energy and $62 \mathrm{eV}$ energy loss. $\mathrm{CoS}$ is an octahedral $\mathrm{Co}^{2+}$ system like $\mathrm{CoO}$. However, in contrast to $\mathrm{CoO}, \mathrm{CoS}$ has only one visible structure in the preedge region of the 1s3p RIXS plane. $\mathrm{CoS}_{2}$ is also a formally divalent sulfide. The RIXS map shows two pre-edge peaks at, respectively, 7710 and $7712 \mathrm{eV}$, diagonally offset. Similar to the case of $\mathrm{Co}_{3} \mathrm{O}_{4}$, these two peaks can be related to the local and 

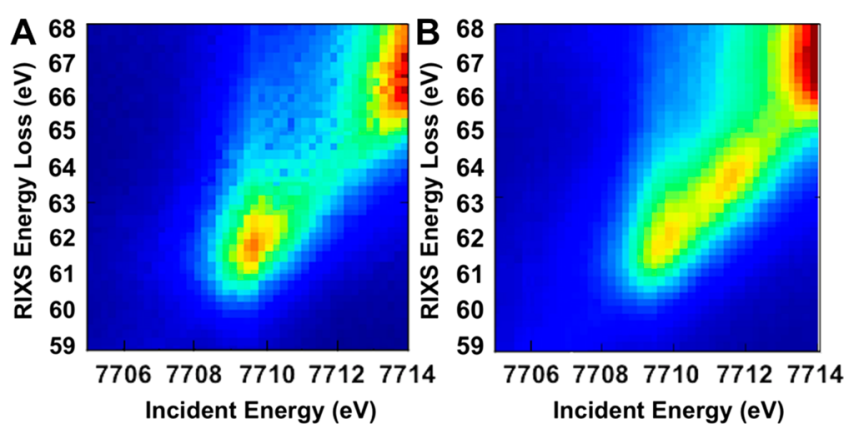

Figure 4. Experimental RIXS 2D contour maps of the CoS (A) and $\mathrm{CoS}_{2}$ (B), measured under ex situ conditions. To prevent oxidation of the sulfide compounds, they were measured under argon atmosphere.

nonlocal transitions. This implies that the $\mathrm{S}_{2}{ }^{2-}$ states link together two neighboring cobalt ions, allowing good overlap between cobalt $4 \mathrm{p}$ orbitals with cobalt $3 \mathrm{~d}$ orbitals at neighboring sites. ${ }^{25}$

3.3. 1s3p RIXS Energy Loss Cuts and the Nonresonant 1s3p XES Spectra. To allow a closer comparison with theoretical simulations, we focus on the RIXS energy loss cuts through the $2 \mathrm{D}$ RIXS plane at the pre-edge maxima. We compare these energy loss cuts with nonresonant XES spectra and also with multiplet calculations.

Figure 5 shows the vertical energy loss cuts through the RIXS plane at the maxima of the pre-edge region for $\mathrm{CoO}$ and

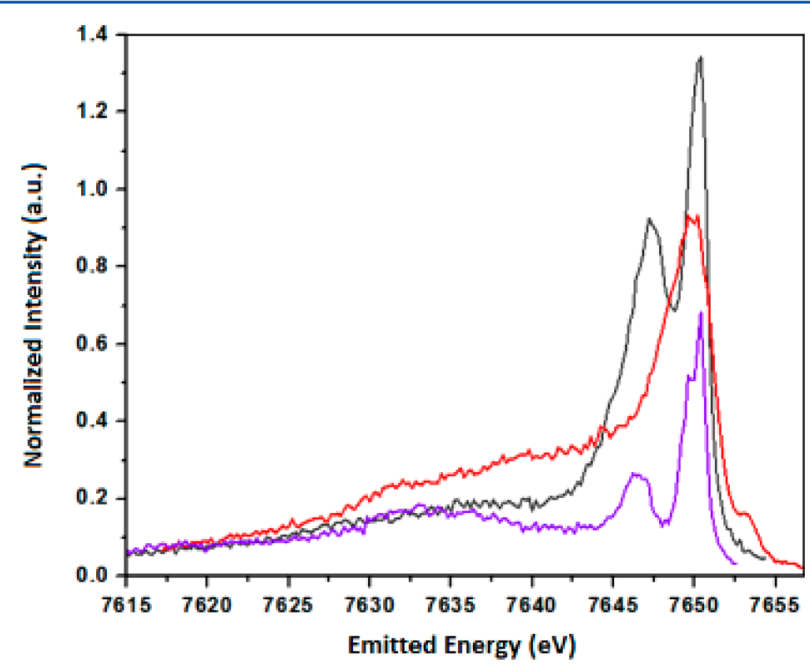

Figure 5. Co $1 \mathrm{~s} 3 \mathrm{p}$ resonant $\mathrm{X}$-ray emission spectra obtained at the pre-edge peak incident energies of $\mathrm{Co}_{3} \mathrm{O}_{4}$ at $7709.4\left(\mathrm{Co}^{2+} T_{\mathrm{d}}\right.$, black $)$ and $7712.2 \mathrm{eV}$ (nonlocal $\mathrm{Co}^{3+} \mathrm{O}_{\mathrm{h}}$, red) and of $\mathrm{CoO}$ at $7709.0 \mathrm{eV}$ $\left(\mathrm{Co}^{2+} \mathrm{O}_{\mathrm{h}}\right.$, purple).

$\mathrm{Co}_{3} \mathrm{O}_{4}$. We have used these energy loss cuts to make a comparison with multiplet calculations. Both the divalent cobalt ions in $\mathrm{CoO}$ and $\mathrm{Co}_{3} \mathrm{O}_{4}$ contain two peaks split by $\sim 2.5 \mathrm{eV}$. The nonlocal $\mathrm{Co}^{3+}$ peak at $7712 \mathrm{eV}$ excitation energy has its main X-ray emission peak at $7649 \mathrm{eV}$. However, due to their shorter $3 \mathrm{p}$ final state lifetime, the low intensity features between 7625 and $7640 \mathrm{eV}$ are broadened. ${ }^{17}$

Figure 6 shows the experimental and calculated cobalt 1s3p RIXS energy loss cuts of CoO. The calculations, have been performed for transitions originated from the $3 \mathrm{~d}^{7}$ ground state via the $1 s^{1} 3 d^{8}$ intermediate states into the $3 p^{5} 3 d^{8}$ final states. The energy loss cut through the $2 \mathrm{D}$ RIXS plane at the main

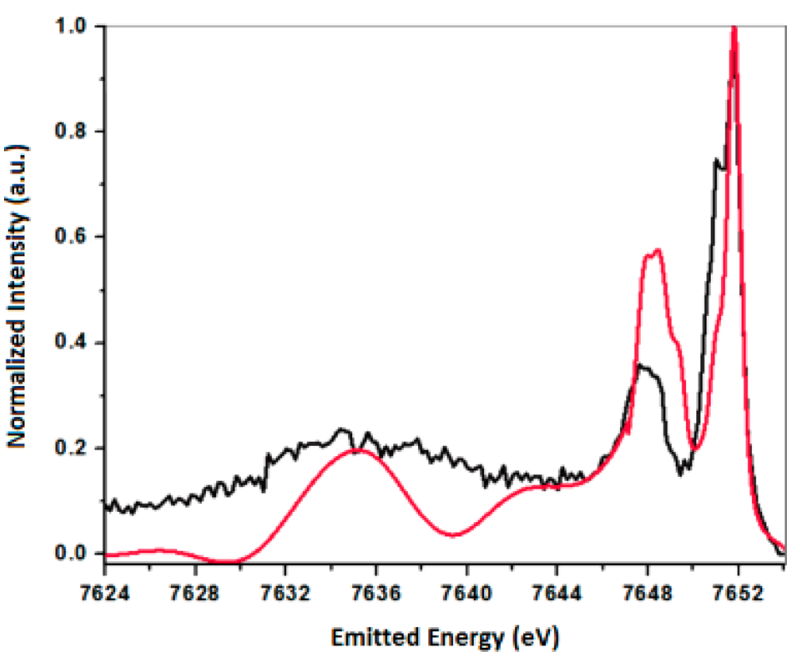

Figure 6. Co 1s3p RIXS energy loss cut of the $\mathrm{CoO}$ sample (black) is compared with the calculated multiplet spectrum (red).

pre-edge peak reproduces the two main emission peaks at 7651.8 and $7647.6 \mathrm{eV}$. Due to the energy-dependent lifetime broadening, all features at higher energies are only visible as broad structures. ${ }^{30}$ The calculations for $\mathrm{Co}^{2+} \mathrm{O}_{\mathrm{h}}$ have been performed with Slater integral reduction factors of $1.0\left(\mathrm{~F}_{\mathrm{dd}}\right), 0.9$ $\left(F_{p d}\right)$, and $0.9\left(G_{p d}\right)$ in combination with a crystal field parameter $10 \mathrm{Dq}$ of 1.0 for the initial and $0.95 \mathrm{eV}$ for the intermediate and final states. Due to the energy dependence of lifetime in case of $\mathrm{K} \beta$ (1s3p) transition the experimental spectrum differs from the multiplet calculated result at lower emission energies. In addition, the diagonal of the obtained $1 \mathrm{~s} 3 \mathrm{p}$ energy loss RIXS plane yields the so-called HERFD spectrum which resembles a high resolution K-edge spectrum.

Figure 7 shows the $\mathrm{Co}_{3} \mathrm{O}_{4}$ 1s $3 \mathrm{p}$ RIXS spectrum in comparison with the corresponding multiplet calculations of the transitions of $\mathrm{Co}^{2+}$ in tetrahedral symmetry. The theoretical calculations for $\mathrm{Co}^{2+} \mathrm{O}_{\mathrm{h}}$ have been performed with Slater integral reduction values of $1.0\left(\mathrm{~F}_{\mathrm{dd}}\right), 0.9\left(\mathrm{~F}_{\mathrm{pd}}\right)$, and $0.9\left(\mathrm{G}_{\mathrm{pd}}\right)$. Furthermore, a crystal field parameter $10 \mathrm{Dq}$ of $-0.6 \mathrm{eV}$ was used to simulate the tetrahedral symmetry.

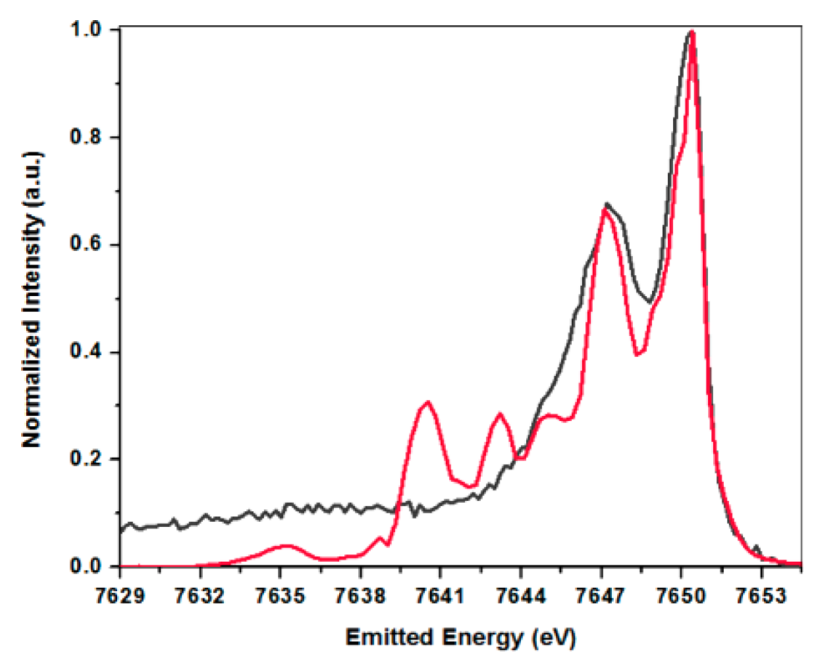

Figure 7. Co 1s3p RIXS energy loss cut of the $\mathrm{Co}_{3} \mathrm{O}_{4}$ sample (black) is compared with the calculated multiplet spectrum. The experimental RIXS spectrum was obtained by plotting the slice $(7709.4 \mathrm{eV}$ incident energy), which has dominantly a $\mathrm{Co}^{2+} T_{\mathrm{d}}$ character. 
In the case of Co RIXS, a core electron is excited to empty $3 \mathrm{~d}$ states and in tetrahedral symmetry also to $4 \mathrm{p}$ states hybridized to $3 \mathrm{~d}$ states. For the quadrupole transition, the X-ray absorption sum rule applies and the intensity ratio of $\mathrm{Co}^{3+} /$ $\mathrm{Co}^{2+}$ is different from nonresonant XES. The total quadrupole intensity is given by the number of $3 \mathrm{~d}$ holes multiplied by the number of ions present, which yields a relative value of 8 (2 ions times 4 holes) for $\mathrm{Co}^{3+}$ and 3 ( 1 ion times 3 holes) is for $\mathrm{Co}^{2+}$. However, as a consequence of $\mathrm{p}-\mathrm{d}$ orbital mixing, the intensity of tetrahedral $\mathrm{Co}^{2+}$ is increased with a factor of approximately four. This implies that the overall $\mathrm{Co}^{3+} / \mathrm{Co}^{2+}$ intensity ratio is approximately $8: 12$. By analyzing the pre-edge RIXS feature we can assign the two features at $7709.4 \mathrm{eV}$ of incident energy for $66 \%$ to the $\mathrm{Co}^{2+} T_{\mathrm{d}}$ ion. From Figure 7, the slice at $7709.4 \mathrm{eV}$ was selected and compared to a $\mathrm{Co}^{2+} T_{\mathrm{d}}$ simulation.

To calculate the RIXS of CoS a ligand field splitting of $10 \mathrm{Dq}$ $=0.4 \mathrm{eV}$ was assumed with Slater integrals scaled down to $70 \%$ $\left(F_{\mathrm{dd}}\right)$, and $50 \%$ (for $\mathrm{F}_{\mathrm{pd}}$ and $\mathrm{G}_{\mathrm{pd}}$ ) from their atomic values to account for the increased covalence of $\mathrm{CoS}$ with respect to the oxides. The RIXS spectrum was obtained from the CTM4RIXS program by selecting a Lorentzian broadening of $1.0 \mathrm{eV}$ for the intermediate state and 0.4 for the final state, while keeping a resolution of $0.3 \mathrm{eV}$ for both the incident and emitted energies.

It can be observed from Figure 8 that, although $\mathrm{CoS}$ is a high-spin $\mathrm{Co}^{2+}$ system, the strong reduction of the Slater

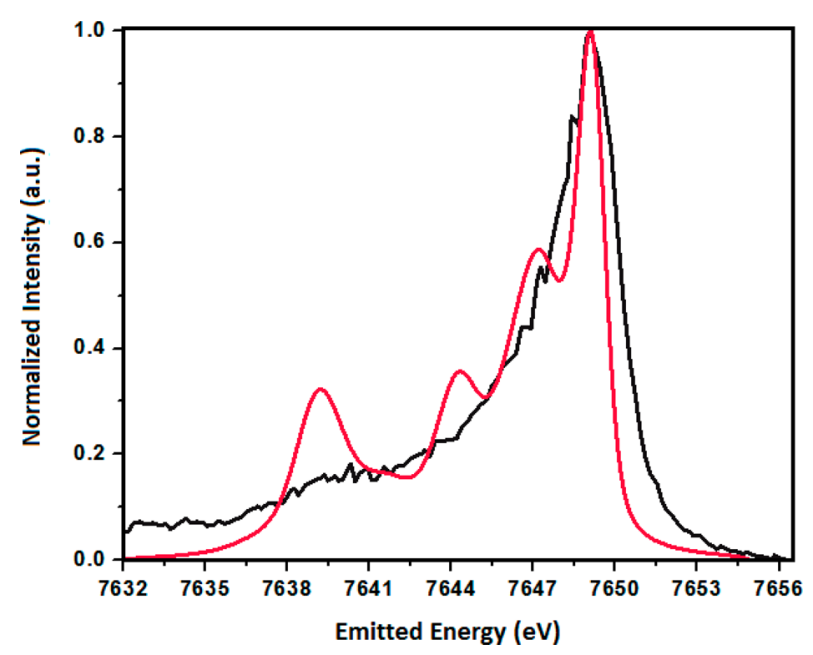

Figure 8. Co 1s3p RIXS energy loss cut of the CoS sample (black) is compared with the calculated multiplet spectrum.

integrals effectively creates a single peak with an asymmetric tail ranging over some $15 \mathrm{eV}$. This is different from the high-spin $\mathrm{Co}^{2+}$ oxide system. The main difference between $\mathrm{CoS}$ and $\mathrm{CoO}$ is the significant increase of covalence. The present calculations only involved crystal field multiplet effects with reduced Slater integrals. A similar spectral shape can be obtained from charge transfer multiplet calculations, but the lack of fine structure and the increased number of charge transfer parameters imply that it is not well justified to use a specific charge transfer multiplet result. ${ }^{31,32}$ A consequence of this increased covalence in $\mathrm{CoS}$ is that the 1s3p RIXS cannot be used to distinguish high-spin and low-spin ground states. Note that because $\mathrm{CoS}_{2}$ is dominated by nonlocal effects we cannot directly compare the energy loss cuts to a calculation

3.4. Fitting of the Two-Dimensional RIXS Planes. To obtain integrated intensities of the spectral features in the RIXS
2D maps of $\mathrm{CoO}$ and $\mathrm{Co}_{3} \mathrm{O}_{4}$, shown in Figure 9, we have performed a two-dimensional surface fitting procedure that involved the methodology implemented in Blueprint XAS to reduce bias and to evaluate the uncertainties in the fit parameters. $^{33,34}$ The empirical model used contains a baseline surface, an edge jump in the incident-energy direction, two $(\mathrm{CoO})$ or three $\left(\mathrm{Co}_{3} \mathrm{O}_{4}\right)$ two-dimensional pre-edge peaks, and three two-dimensional $\mathrm{K} \beta$ peaks in each case. A series of 100 fits have been attempted and a series of good fits based on sum of squared errors was selected for the quantification of the preedge features in each case. The average for the intensities and their respective standard deviation values are summarized in Table 1. The average of fits for each RIXS map is shown in Figure 9.

Table 1 shows that $\mathrm{CoO}$ contains two peaks at almost the same excitation energy (shift of $0.6 \mathrm{eV}$ ) and split by $3.6 \mathrm{eV}$ in the emission energy. Although the first peak has a higher intensity in the energy loss cut (Figure 6), their integrated intensities are similar in magnitude due to the large broadenings of the second peak. In the case of $\mathrm{Co}_{3} \mathrm{O}_{4}$ two similar peaks appear but with increased intensity due to the tetrahedral-symmetry-induced $\mathrm{p}-\mathrm{d}$ mixing. In addition, the $62.0 \mathrm{eV}$ energy transfer feature contains additional intensity due to the overlapping $\mathrm{Co}^{3+}$ quadrupole peak. The third peak at $7712 \mathrm{eV}$ excitation energy is the $\mathrm{Co}^{3+}$ nonlocal peak.

\section{CONCLUDING REMARKS}

We have identified the electronic properties of cobalt oxide and sulfide compounds by means of $1 \mathrm{~s} 3 \mathrm{p}$ RIXS. High resolution RIXS applied to cobalt oxide and sulfide compounds revealed details of the pre-edge features, which are not visible in conventional XAS spectroscopy. The results were simulated by multiplet calculations with the associated parameters for the symmetry, crystal field, and Slater Integral reduction.

The CoO 1s3p RIXS plane consists of two features at the $7709.0 \mathrm{eV}$ incident energy related to $\mathrm{Co}^{2+}\left(\mathrm{O}_{\mathrm{h}}\right)$. Instead, the $\mathrm{Co}_{3} \mathrm{O}_{4}$ 1s $3 \mathrm{p}$ RIXS plane consists of two features at $7709.6 \mathrm{eV}$ incident energy related mainly to $\mathrm{Co}^{2+}\left(T_{\mathrm{d}}\right)$ and a third feature at the $7712 \mathrm{eV}$ incident energy that was assigned as a nonlocal transition at the $\mathrm{Co}^{3+}\left(\mathrm{O}_{\mathrm{h}}\right)$ site. The $\mathrm{Co}^{3+}$ quadrupole peak is not visible as a separate feature and is buried under the $\mathrm{Co}^{2+}$ $\left(T_{\mathrm{d}}\right)$ spectrum. It can be concluded that the nonlocal peak is also clearly visible in the spectra of $\mathrm{Co}_{3} \mathrm{O}_{4}$, similar to other lowspin trivalent cobalt oxides, such as $\mathrm{LaCoO}_{3}$ and $\mathrm{LiCoO}_{2}{ }^{27,35}$

An interesting result for the cobalt sulfides is that, in contrast to $\mathrm{CoS}, \mathrm{CoS}_{2}$ shows a strong nonlocal peak, indicating significant sulfur-mediated cobalt-cobalt overlap, similar to the case of the low-spin trivalent oxides. This indicates that though the cobalt ions in $\mathrm{CoS}_{2}$ are indicated as $\mathrm{Co}^{2+}$, they have a much stronger sulfur-mediated cobalt-cobalt overlap as compared with CoS.

Another important result for the cobalt sulfides is that, due to the strong covalency, the $3 \mathrm{p} 3 \mathrm{~d}$ exchange interaction is strongly screened, implying that the $3 p$ final state contains only a single asymmetric peak, in contrast to the double peak structure in high-spin oxides. A consequence is that the 1s3p XES spectra cannot distinguish high-spin and low-spin systems from a qualitative analysis of the spectral shape.

This study has shown that $1 \mathrm{~s} 3 \mathrm{p}$ RIXS is a valuable tool to determine the valence and covalence and estimate the spin state of cobalt oxides and sulfides, with the exception that for sulfides the large covalence does not allow a distinction between highspin and low-spin. The obtained knowledge can be used to 

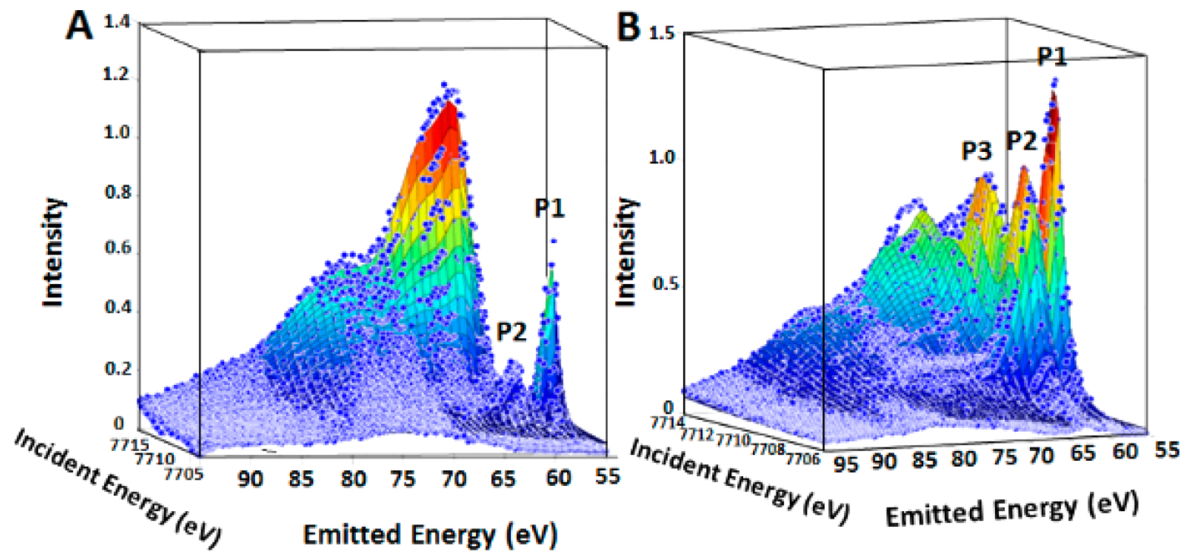

Figure 9. Average of fits to the experimental two-dimensional 1s3p-RIXS maps of (A) $\mathrm{CoO}$ and (B) $\mathrm{Co}_{3} \mathrm{O}_{4}$. Pre-edge features (peaks 1 and 2) and nonlocal feature (peak 3) are indicated for reference in Table 1.

Table 1. Average Values for the Fit Intensities and Corresponding Standard Deviations for the Pre-Edge Features in the 1s3p RIXS 2D Maps of $\mathrm{Co}_{3} \mathrm{O}_{4}$ and $\mathrm{CoO}^{a}$

\begin{tabular}{|c|c|c|c|c|c|}
\hline & $\begin{array}{c}\text { incident } \\
\text { energy } \\
(\mathrm{eV})\end{array}$ & $\begin{array}{l}\text { energy } \\
\text { loss } \\
(\mathrm{eV})\end{array}$ & $\begin{array}{l}\text { HWHM } \\
\text { (incident; } \\
\text { eV) }\end{array}$ & $\begin{array}{l}\text { HWHM } \\
\text { (energy loss; } \\
\text { eV) }\end{array}$ & $\begin{array}{l}\text { integrated } \\
\text { intensity }\end{array}$ \\
\hline \multicolumn{6}{|l|}{$\mathrm{Co}_{3} \mathrm{O}_{4}$} \\
\hline peak 1 & $\begin{array}{l}7709.5 \\
(<0.1)\end{array}$ & $\begin{array}{l}59.2 \\
(<0.1)\end{array}$ & $\stackrel{0.68}{(<0.001)}$ & $0.66(0.001)$ & $\begin{array}{r}3.21 \\
(0.02)\end{array}$ \\
\hline peak 2 & $\begin{array}{l}7709.7 \\
\quad(<0.1)\end{array}$ & $\begin{array}{l}62.0 \\
(<0.1)\end{array}$ & $\begin{array}{l}0.91 \\
(0.001)\end{array}$ & $1.99(0.01)$ & $\begin{array}{c}13.04 \\
(0.11)\end{array}$ \\
\hline peak 3 & $\begin{array}{r}7712.2 \\
(<0.1)\end{array}$ & $\begin{array}{l}62.4 \\
(<0.1)\end{array}$ & $1.23(0.01)$ & $1.45(0.02)$ & $\begin{array}{l}5.81 \\
(0.11)\end{array}$ \\
\hline \multicolumn{6}{|l|}{$\mathrm{CoO}$} \\
\hline peak 1 & $\begin{array}{l}7709.0 \\
(<0.1)\end{array}$ & $\begin{array}{l}57.5 \\
(<0.1)\end{array}$ & $\begin{array}{l}0.71 \\
\quad(0.002)\end{array}$ & $0.75(<0.001)$ & $\begin{array}{l}1.57 \\
(0.02)\end{array}$ \\
\hline peak 2 & $\begin{array}{l}7709.6 \\
(<0.1)\end{array}$ & $\underset{(<0.1)}{61.1}$ & $1.23(0.04)$ & $2.18(0.04)$ & $\begin{array}{l}1.77 \\
(0.09)\end{array}$ \\
\hline
\end{tabular}

${ }^{a}$ Standard deviations are given in parentheses.

understand and describe the properties of cobalt materials in the field of, for example, catalysis and allows us to study functional materials under in situ conditions.

\section{AUTHOR INFORMATION}

\section{Corresponding Author}

*E-mail: f.m.f.degroot@uu.nl.

\section{Notes}

The authors declare no competing financial interest.

\section{ACKNOWLEDGMENTS}

This research is performed with financial support from STWNWO (Netherlands Organization for Scientific Research, with Grant No. NANO-11507). The experiments at SPring-8 were performed under the approvals of the Japan Synchrotron Radiation Research Center (Proposal No. 2014A4255, 2014B4262) and the National Synchrotron Radiation Research Center, Taiwan (Proposal No. 2014-2-086, 2014-3-072). We acknowledge the SOLEIL Synchrotron for provision of the synchrotron radiation facilities (Proposal No. 99140069).

\section{REFERENCES}

(1) Schmitt, T.; de Groot, F. M. F.; Rubensson, J. Prospects of Highresolution Resonant X-ray Inelastic Scattering Studies on Solid Materials, Liquids and Gases at Diffraction-limited Storage Rings. J. Synchrotron Radiat. 2014, 21, 1065-1076.
(2) Caracas, R.; Ozawa, H.; Hirose, K.; Ishii, H.; Hiraoka, N.; Ohishi, Y.; Hirao, N. Identifying the Spin Transition in $\mathrm{Fe}^{2+}$-rich $\mathrm{MgSiO}_{3}$ Perovskite from X-ray Diffraction and Vibrational Spectroscopy. Am. Mineral. 2014, 99, 1270-1276.

(3) de Groot, F. High-resolution X-ray Emission and X-ray Absorption Spectroscopy. Chem. Rev. 2001, 101, 1779-1808.

(4) Griffith, J. S. The Theory of Transition Metal Ions; University Press: Cambridge, 1964.

(5) Westre, T. E.; Kennepohl, P.; DeWitt, J. G.; Hedman, B.; Hodgson, K. O.; Solomon, E. J. Am. Chem. Soc. 1997, 119, 62976314.

(6) Kortright, J. B.; Thompson, A. C. X-ray Data Booklet; Thompson, A. C., Vaughan, D., Eds.; Lawrence Berkeley National Laboratory, 2001.

(7) Tsutsumi, K.; Nakamori, H.; Ichikawa, K. X-ray Mn K $\beta$ Emission Spectra of Manganese oxides and Manganates. Phys. Rev. B 1976, 13, 929-933.

(8) Hämäläinen, K.; Siddons, D.; Hastings, J.; Berman, L. Elimination of the Inner-Shell Lifetime Broadening in X-Ray Absorption Spectroscopy. Phys. Rev. Lett. 1991, 67, 2850-2853.

(9) de Groot, F.; Krisch, M.; Vogel, J. Spectral Sharpening of the Pt L edges by High-resolution X-ray Emission. Phys. Rev. B: Condens. Matter Mater. Phys. 2002, 66, 195112.

(10) Kurian, R.; van Schooneveld, M. M.; Zoltan, N.; Vankó, G.; de Groot, F. M. F. Temperature Dependent 1s2p Resonant Inelastic XRay Scattering of CoO. J. Phys. Chem. C 2013, 117, 2976-2981.

(11) Delmon, B. New Technical Challenges and Recent Advances in Hydrotreating Catalysis. A Critical updating Review. Catal. Lett. 1993, $22,1-32$.

(12) Petitto, S. C.; Marsh, E. M.; Carson, G. A.; Langell, M. Cobalt oxide Surface Chemistry: The Interaction of $\mathrm{CoO}\left(\begin{array}{lll}1 & 0 & 0\end{array}\right), \mathrm{Co}_{3} \mathrm{O}_{4}\left(\begin{array}{lll}1 & 1\end{array}\right.$ $0)$, and $\mathrm{Co}_{3} \mathrm{O}_{4}\left(\begin{array}{lll}1 & 1 & 1\end{array}\right)$ with Oxygen and Water. J. Mol. Catal. A: Chem. 2008, 281, 49-58.

(13) Greenwald, S. The Antiferromagnetic Structure Deformations in $\mathrm{CoO}$ and MnTe. Acta Crystallogr. 1953, 6, 396-398.

(14) Smith, W.; Hobson, A. The Structure of Cobalt oxide, $\mathrm{Co}_{3} \mathrm{O}_{4}$. Acta Crystallogr., Sect. B: Struct. Crystallogr. Cryst. Chem. 1973, 29, 362-363.

(15) Cai, Y. Q.; Chow, P.; Chen, C. C.; Ishii, H.; Tsang, K. L.; Kao, C. C.; Liang, K. S.; Chen, C. T. Optical Design and Performance of the Taiwan Inelastic X-Ray Scattering Beamline (BL12XU) at SPring-8. AIP Conf. Proc. 2003, 705, 340-343.

(16) Rueff, J.-P.; Ablett, J. M.; Céolin, D.; Prieur, D.; Moreno, T.; Balédent, V.; Lassalle-Kaiser, B.; Rault, J. E.; Simon, M.; Shukla, A. The Galaxies Beamline at Soleil Synchrotron: Inelastic X-ray Scattering and Photoelectron Spectroscopy in the Hard X-ray Range. J. Synchrotron Radiat. 2015, 22, 175-179.

(17) de Groot, F. M. F.; Kotani, A. Core Level Spectroscopy of Solids; Taylor \& Francis: New York, 2008. 
(18) Westre, T. E.; Kennepohl, P.; De Witt, J. G.; Hedman, B.; Hodgson, K. O.; Solomon, E. I. A Multiplet Analysis of Fe K-edge $1 \mathrm{~s} \rightarrow 3 \mathrm{~d}$ Pre-edge Features of Iron Complexes. J. Am. Chem. Soc. 1997, 119, 6297-6314.

(19) de Groot, F. High-resolution X-ray Emission and X-ray Absorption Spectroscopy. Chem. Rev. 2001, 101, 1779-1808.

(20) Arrio, M.; Rossano, S.; Brouder, C.; Galoisy, L.; Calas, G. Calculation of Multipole Transitions at the Fe K Pre-edge Through pd hybridization in the Ligand Field Multiplet Model. Europhys. Lett. 2000, 51, 454-460.

(21) Thole, B.; Cowan, R.; Sawatzky, G.; Fink, J.; Fuggle, J. New Probe for the Ground State Electronic Structure of Narrow Band and Impurity States. Phys. Rev. B: Condens. Matter Mater. Phys. 1985, 31, 6856-6858.

(22) de Groot, F.; Fuggle, J.; Thole, B.; Sawatzky, G. 2p X-ray Absorption of 3d Transition-metal Compounds: An Atomic Multiplet Description Including the Crystal Field. Phys. Rev. B: Condens. Matter Mater. Phys. 1990, 42, 5459-5468.

(23) Kotani, A.; Shin, S. Resonant Inelastic X-ray Scattering Spectra for Electrons in Solids. Rev. Mod. Phys. 2001, 73, 203-246.

(24) Stavitski, E.; de Groot, F. M. F. The CTM4XAS Program for EELS and XAS Spectral Shape Analysis of Transition Metal L edge. Micron 2010, 41, 687-694.

(25) van Schooneveld, M. M.; Kurian, R.; Juhin, A.; Zhou, K.; Schlappa, J.; Strocov, V. N.; Schmitt, T.; de Groot, F. M. F. A Multispectroscopic Study of 3d Orbitals in Cobalt Carboxylates: The High Sensitivity of 2p3d Resonant X-ray Emission Spectroscopy to the Ligand Field. J. Phys. Chem. C 2012, 116, 15218-15230.

(26) Vankó, G.; Rueff, J.-P.; Mattila, A.; Németh, Z.; Shukla, A. Temperature-and pressure-induced spin-state transitions in $\mathrm{LaCoO}_{3}$. Phys. Rev. B: Condens. Matter Mater. Phys. 2006, 73, 024424.

(27) Juhin, A.; de Groot, F.; Vankó, G.; Calandra, M.; Brouder, C. Angular Dependence of Core hole Screening in $\mathrm{LiCoO}_{2}$ : A DFT+ U Calculation of the Oxygen and Cobalt K-edge X-ray Absorption Spectra. Phys. Rev. B: Condens. Matter Mater. Phys. 2010, 81, 115115.

(28) Shukla, A.; Calandra, M.; Taguchi, M.; Kotani, A.; Vankó, G.; Cheong, S.-W. Polarized Resonant Inelastic X-ray Scattering as an Ultrafine Probe of Excited States of $\mathrm{La}_{2} \mathrm{CuO}_{4}$. Phys. Rev. Lett. 2006, 96, 077006.

(29) Vankó, G.; de Groot, F. M. F.; Huotari, S.; Cava, R. J.; Lorenz, Th.; Reuther, M. Intersite 4p-3d Hybridization in Cobalt oxides: A Resonant X-ray Emission Spectroscopy Study. arXiv:0802.2744v1 2008.

(30) Hayashi, H.; Azumi, T.; Sato, A.; Udagawa, Y. A Cartography of $\mathrm{K} \beta$ Resonant Inelastic X-ray Scattering for Lifetime-broadeningsuppressed Spin-selected XANES of $\alpha-\mathrm{Fe}_{2} \mathrm{O}_{3}$. J. Electron Spectrosc. Relat. Phenom. 2008, 168, 34-39.

(31) Glatzel, P.; Mirone, A.; Eeckhout, S. G.; Sikora, M.; Giuli, G. Orbital Hybridization and Spin Polarization in the Resonant $1 \mathrm{~s}$ Photoexcitations of $\alpha-\mathrm{Fe}_{2} \mathrm{O}_{3}$. Phys. Rev. B: Condens. Matter Mater. Phys. 2008, 77, 115133 .

(32) Glatzel, P.; Bergmann, U.; de Groot, F.; Cramer, S. Influence of the Core hole on Kbeta Emission Following Photoionization or Orbital Electron Capture: A Comparison using $\mathrm{MnO}$ and ${ }^{55} \mathrm{Fe}_{2} \mathrm{O}_{3}$. Phys. Rev. B: Condens. Matter Mater. Phys. 2001, 64, 045109.

(33) Delgado-Jaime, M. U.; Mewis, C. P.; Kennepohl, P. Blueprint XAS: a Matlab-based Toolbox for the Fitting and Analysis of XAS Spectra. J. Synchrotron Radiat. 2010, 17, 132-137.

(34) Delgado-Jaime, M. U.; Kennepohl, P. Development and Exploration of a New Methodology for the Fitting and Analysis of XAS Data. J. Synchrotron Radiat. 2010, 17, 119-128.

(35) de Groot, F.; Vankó, G.; Glatzel, P. The 1s X-ray Absorption Pre-edge Structures in Transition Metal Oxides. J. Phys.: Condens. Matter 2009, 21, 104207. 